\title{
Comparison between proportional, integral, derivative controller and fuzzy logic approaches on controlling quarter car suspension system
}

\author{
Ahmet Mehmet Karadeniz ${ }^{1,{ }^{*}}$, Ammar Alsabbagh ${ }^{1}$, and Dr.Geza Husi ${ }^{1}$ \\ ${ }^{1}$ University of Debrecen, Department of Mechatronics, 4028 Debrecen Otemeto utca 2.-4., Hungary
}

\begin{abstract}
Developing and constantly changing technologies, efforts to achieve maximum efficiency with minimum fuel consumption, as well as the development of comfort and safety systems, have become very essential topic in car manufacturing and design. Whereas comfort and security were not given a high importance in the first produced cars, they are indispensable elements of today's automobiles. Since public transportation uses road in large scale, the need for safety and repose is also increasing. Nowadays, vehicles have better security and comfort systems, which react very quickly to all kinds of loads and different cases of driving (braking, acceleration, high speed, cornering), where the tires can keep the road at its best, utilizing an advanced suspension system. In this study, a quarter-car model was fulfilled using LabVIEW (Laboratory Virtual Instrumentation Engineering Workbench) software. The control of this model has been realized by applying two different controllers. PID (proportional, integral, derivative) controller which is a common and conventional control method and the Fuzzy Logic controller which is considered as an expert system that is becoming more and more widely used. In both control approaches, controlling the suspension system was achieved successfully. However; It has been determined that controlling the system using Fuzzy Logic controller gave better dynamic response than applying the PID controller for the quarter car suspension model that has been used in the direction of this study.
\end{abstract}

\section{Introduction}

In recent years, improving of vehicle suspension systems has been an intensive research and development topic. These studies are carried out in two different forms, scientific and commercial. Commercial work is carried out by vehicle and parts manufacturers aiming to enhance the quality of their products. Scientific studies are carried out by researchers, it concentrates more on controlling the system in order to provide automatic control of vehicle suspensions relying on the new developments of actuators, sensors and low cost electronics. The features expected from a good suspension system make suspension control more interesting. The desired features are:

- Adjustment of body movement: Ideally, a suspension should protect the body from road irregularities and from irregularities caused by inertia at the start, stop and during cornering.

- Adjustment of the suspension movement: Extreme vertical movement of the wheel will cause the tire to be in an improper position with respect to the road, which will also result a poor roadholding. In other words, the surface between the tires and the road should be maximized in order to increase the engine efficiency.
- Force distribution: Proper tire and road contact must be provided on all four wheels to ensure a good roadholding and handling.

These features overlap each other according to the road and vehicle situation, therefor, traditional suspension systems cannot provide all these features. A design that reduces the sideways tilt of the torso, for instance, will have too much stiffness, which cause the vibrations from the road to be transmitted to the vehicle body.

This can be shown on the quarter car model in which the single wheel is considered. The amplitude of the transfer function, which relates the vertical changes on the curve to the changes in the vertical velocity of the vehicle body, is seen. The straight line is the answer of the traditional vehicle suspension system. There are two resonance states. Lower one; the vibrations of the body (spring mass), and the other one; that is related to the vibrations of the wheel hub (unspring mass). Improving the low frequency characteristics of the suspension may be possible by increasing the damping constant of the suspension. The dotted line indicates the case where the damping constant is increased ten times. The response at low frequencies can be improved by shifting the resonance region. Note that the resonance amplitudes of the body and the wheel increase while the gain at high frequencies decreases.

\footnotetext{
* Corresponding author: ahmetm.karadeniz@gmail.com
} 
The simple quarter car model mentioned above clearly demonstrates the dilemma between low and highfrequency driving comfort. Furthermore, the control of the side tilt of the nose and the rear part will be a function of the interaction between each suspension [1].

\section{Quarter Car Model}

The quarter car model, which is one of the most preferred, most useful and simplest car models, is shown in Fig. 1. The parameters of the model used in this study are obtained by extracting the mathematical model of the system and using the reference [2].

The vibrations of a vehicle subjected to a vertical axis or as result from dynamic behaviours can be modelled as $M_{1}$ and $M_{2}$ double masses. $M_{1}$ represents quarter of the total mass of the vehicle, and $\mathrm{M}_{2}$ represents the mass of the wheel of the vehicle.

The equations for the quarter car suspension model are shown in the both equations 1 and 2 :

$$
\begin{aligned}
& M_{1} \ddot{x}_{1}=-b\left(\dot{x}_{1}-\dot{x}_{2}\right)-K_{1}\left(x_{1}-x_{2}\right)+u \\
& M_{2} \ddot{x}_{2}=-b\left(\dot{x}_{1}-\dot{x}_{2}\right)-K_{1}\left(x_{1}-x_{2}\right)+ \\
& b_{2}\left(\dot{w}-\dot{x}_{2}\right)+k_{2}\left(w-x_{2}\right)-u
\end{aligned}
$$

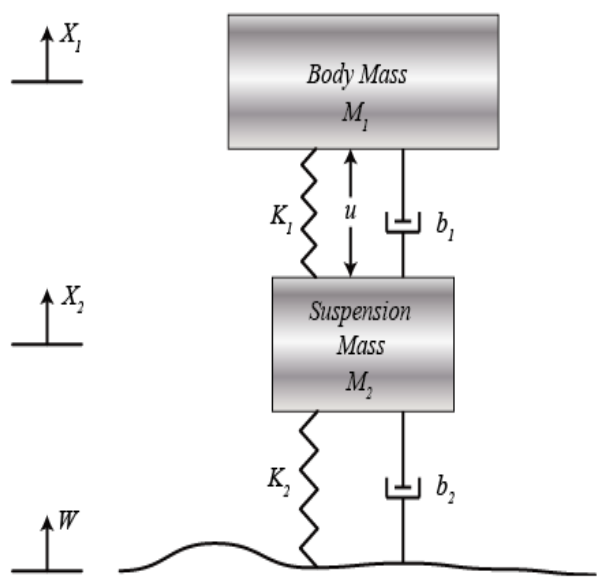

Fig. 1. Quarter Car Suspension System.

\subsection{Modelling Quarter Car Suspension System}

LabVIEW (Laboratory Virtual Instrumentation Engineering Workbench) allows modelling of systems that can be expressed as mathematical equations using block diagrams. The modelling in the LabVIEW environment using the equations for the quarter vehicle suspension model is shown in Fig. 2. The parameters and values used during this modelling are shown in Table 1.

Table 1. System Parameters

\begin{tabular}{|c|cc|}
\hline Parameter & Value & Unit \\
\hline $\mathrm{M}_{1}$ & 2500 & $\mathrm{Kg}$ \\
$\mathrm{M}_{2}$ & 320 & $\mathrm{Kg}$ \\
$\mathrm{K}_{1}$ & 80000 & $\mathrm{~N} / \mathrm{m}$ \\
\hline
\end{tabular}

\begin{tabular}{|c|cc|}
\hline $\mathrm{K}_{2}$ & 500000 & $\mathrm{~N} / \mathrm{m}$ \\
$\mathrm{b}_{1}$ & 350 & $\mathrm{~N} . \mathrm{s} / \mathrm{m}$ \\
$\mathrm{b}_{2}$ & 15020 & N.s $/ \mathrm{m}$ \\
\hline
\end{tabular}

As minimising the vibration is one of this research most significant aims, controlling the vertical movement and forcing it to goes to zero is what is important. For nonfeedback systems, the desired parameter cannot be controlled or only the open-loop response, which is the uncontrolled response of the system, can be obtained. Fig. 3 shows the open loop response obtained for the quarter car suspension system. As shown in Fig. 3, it has an oscillated dumbed behaviour because it has no feedback nor controller (open loop response).

\subsection{Controlling System Utilizing PID}

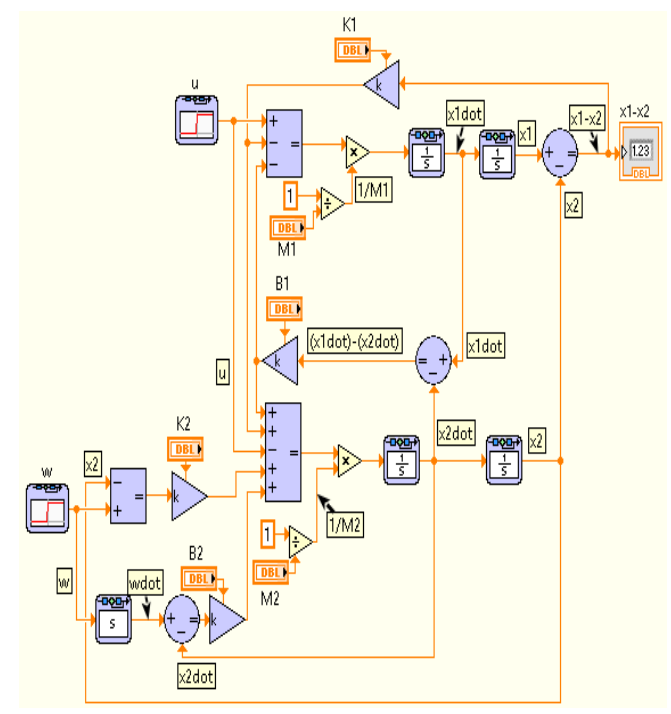

Fig. 2. LabVIEW Model of the System.

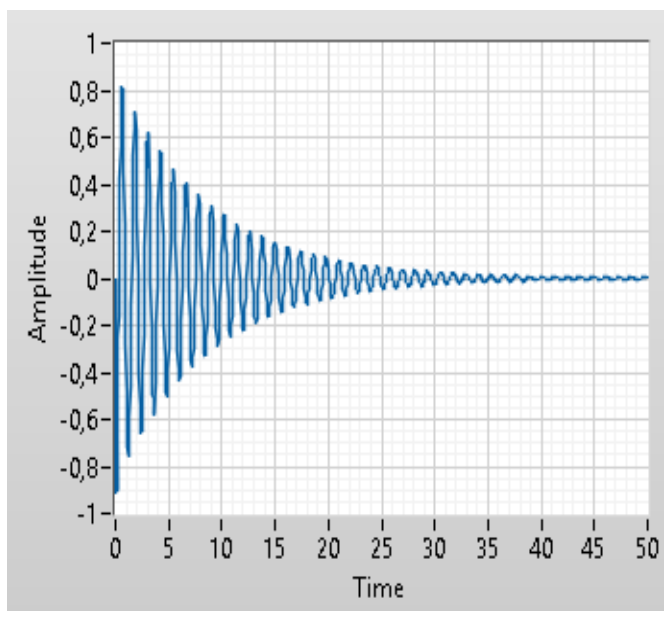

Fig. 3. Open Loop Response of the System.

PID control is a proportional integral plus derivative controller whose transfer function is:

$$
G_{P I D}(s)=K_{p}+\frac{K_{i}}{s}+K_{d} s
$$


PID controller is considered as one of the simplest controllers, easy to be applied and it does not require high performance microprocessors [5]. The PID gains can be obtained using many different methods like zero-pool cancelation, Ziegler-Nichols, and trial and error. In this paper, Trial and error method has been used in order to tune the PID controller. $\quad K_{p}, K_{i}, K_{d}$ gains are called proportional PID gain, integral PID gain, and derivative PID gain, respectively. Error signal is the input for proportional, derivative and integral gains of the PID controller to obtain desired output. The block diagram of the LabVIEW PID controller with the subsystem which represents quarter car suspension system for this study model is shown in Figure 4.

Where the PID (proportional, integral, derivative) controller is applied to the quarter car suspension system as series with a feedback. Table 2 shows the values used for PID parameters which are $K_{p}, K_{i}, K_{d}$ gains.

Table 2. PID Parameters

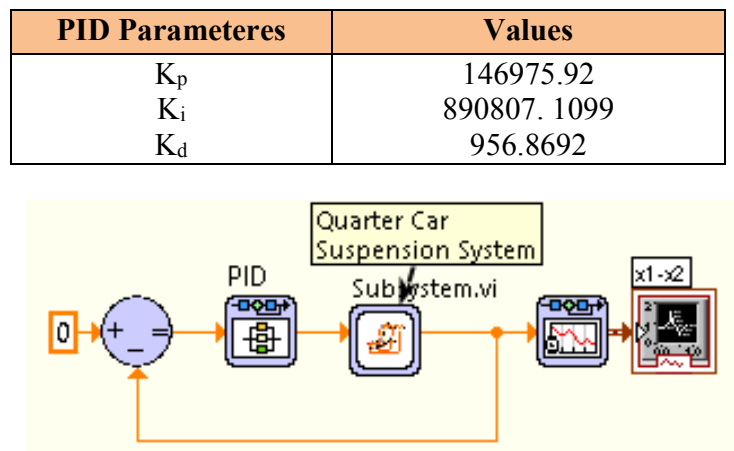

Fig. 4. Controlling the suspension system using PID

Fig. 5 shows the obtained result when the PID controller is applied with the parameters given in Table 2. Looking at this result, we can see that the system respond is zero after 1 second. The settling down of the system to zero means that the vibrations along the vertical axis in the vehicle will not affect the passengers.

\subsection{Controlling System Utilizing Fuzzy Logic}

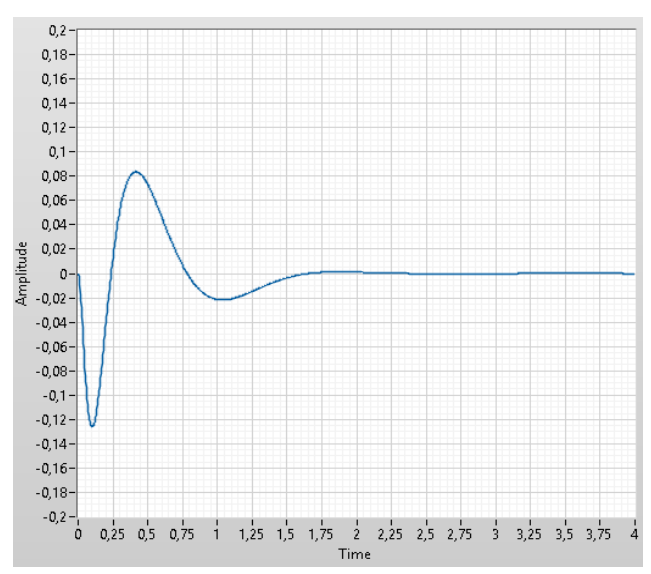

Fig. 5. The system response to the PID controller

Fuzzy logic, unlike classical logic, does not have sharp distinctions. That is, one level does not only have only 0 or 1 values, but it can also have values in between.
NB (negative big), NS (negative small), Z (zero), PS (positive small), PB (positive big) levels, which are commonly used in fuzzy logic controllers. Fuzzy System Designer is used to transfer these levels to the LabVIEW environment. With Fuzzy System Designer, the membership functions of the system were defined using the table 3 , which was created by experts and the values of membership functions can be determined. After the decisions, the control model is designed with the fuzzy logic controller in LabVIEW. The fuzzy logic controller model designed for this system is shown in Fig. 6. Since identification of membership functions the fuzzy logic controller is important, the type, number and normalization factors of the membership functions should be carefully determined at this step. In this study, the general trapezoidal membership function was chosen however, only the $\mathrm{Z}$ membership function was chosen as the triangular membership function because it is desired to be highly sensitive to make the error goes to zero as precise as possible. In the normalization process, the input function is kept in the range of -1 to +1 .

The rule tables that show the relationship between the input and output in the control with the fuzzy logic controller are important factors. The rule table used for this study is shown in Table 3 and membership functions are shown in Fig. 7 on Fuzzy System Designer [3,4].

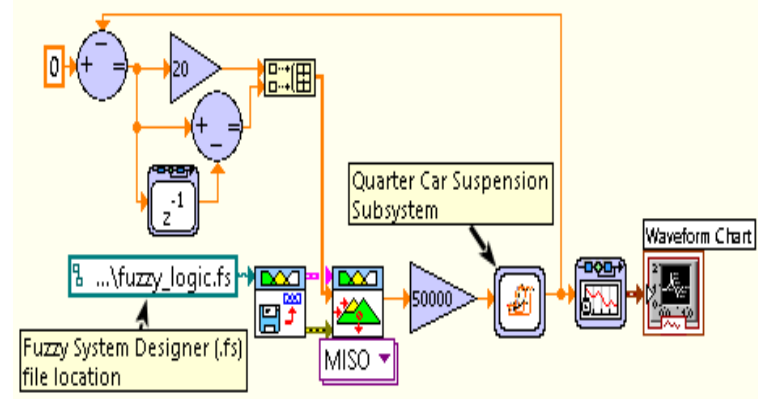

Fig. 6. Controlling the system using Fuzzy Logic

Table 3. Rule Table

\begin{tabular}{|c|ccccc|}
\hline $\boldsymbol{d} \boldsymbol{u} \boldsymbol{u}$ & $\boldsymbol{N} \boldsymbol{B}$ & $\boldsymbol{N} \boldsymbol{S}$ & $\boldsymbol{Z}$ & $\boldsymbol{P} \boldsymbol{S}$ & $\boldsymbol{P} \boldsymbol{B}$ \\
\hline $\boldsymbol{N} \boldsymbol{B}$ & NB & NB & NB & NS & Z \\
$\boldsymbol{N} \boldsymbol{S}$ & NB & NB & NS & Z & PS \\
$\boldsymbol{Z}$ & NB & NB & Z & PS & PB \\
$\boldsymbol{P S}$ & NS & NS & Z & PB & PB \\
$\boldsymbol{P} \boldsymbol{B}$ & Z & Z & PS & PB & PB \\
\hline
\end{tabular}

In order to evaluate the performance of the fuzzy logic controller designed in accordance with the rules and membership functions specified in Table 3 and Fig. 7, it is necessary to compare the output values with the desired values. The closed-loop response of the system controlled using the fuzzy logic controller for this study is demonstrated in Fig. 8.

The choosing of the controller to be applied in order to control any system is performed as a result of comparing the responses of the specified controllers to this system. Fig. 9 shows the response of both controllers for this system.

The behaviour of the vehicle's suspension system without any controller is shown in the open loop response in Fig. 3. To conclude from this, the uncontrolled vehicle 

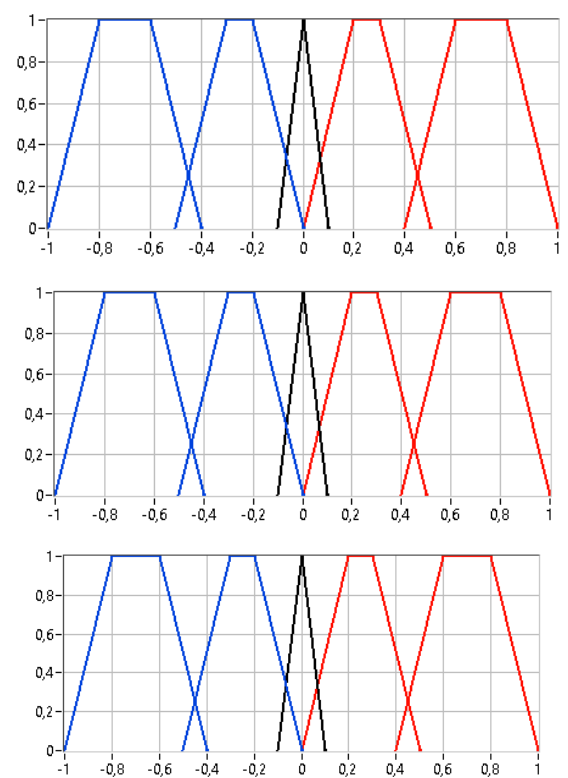

Fig. 7. Membership Functions 'u', ' $\Delta u$ ', 'output'.

suspension system is not desirable considering material life and passenger health and safety. A control method

\subsection{Comparison of PID and Fuzzy Logic}

should be selected to remove such undesirable situations and developed in such a way that the control parameters are applied to the system to provide the optimum level. The control method that should be applied to the quarter car suspension system will be clarified by comparing the results obtained in this study.

As can be seen form fig. 9, the response using fuzzy logic controller is tree time less downshoot than the utilizing PID (about 4 centimetres downshoot utilizing fuzzy logic controller against 13 centimetres downshoot using PID controller). Moreover, control the quarter suspension system takes about half of consumed time by PID to reach the steady state (the fuzzy logic controller leaded the system to reach the stable state in approximately 0.75 second, while the PID controller took around 1.5 seconds to make the quarter suspension system reach the steady state).

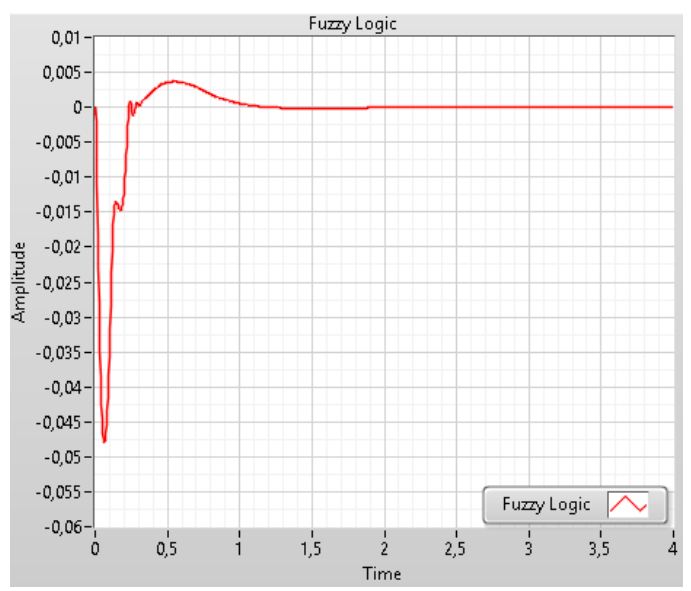

Fig. 8. The system response to Fuzzy Logic controller

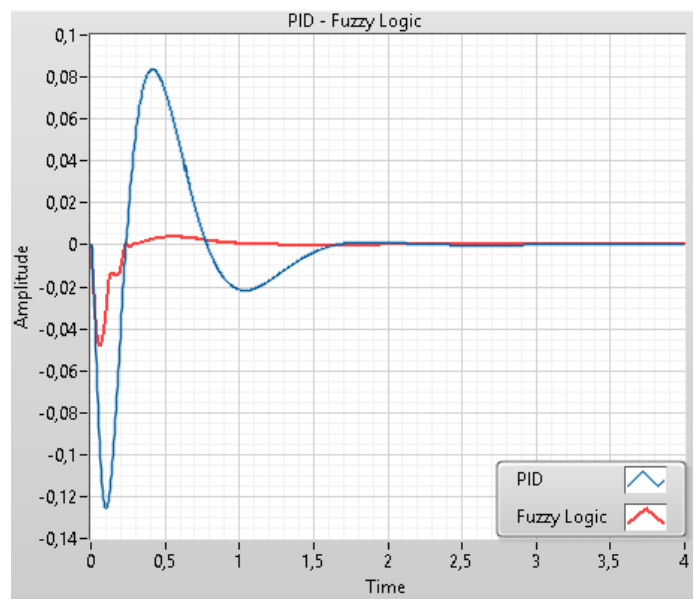

Fig. 9. Comparison of PID and Fuzzy Logic Controllers.

\section{Conclusions}

The performance of the PID and Fuzzy Logic controllers for the quarter car suspension system was tested. The model used in this study is taken from [1]. Fuzzy Logic and PID controllers were applied to the system separately and the obtained results were compared with each other. In this comparison, it has been observed that the Fuzzy Logic controller gives better results than the PID controller for the control of the designed model in respect of both time that was taking to reach the steady state, and in respect of overshoot and downshoot, which means, that the fuzzy logic controller was able to reduce the oscillation amplitude of the system and enhance the safety of mechanical parts of the vehicle as well as the passenger safety.

\section{Acknowledgment}

The publication is supported by the EFOP-3.6.1-16-201600022 project. The project is co-financed by the European Union and the European Social Fund. Moreover, thanks to Adewale Oriyomi Oseni for being encouraging, supporting, and making this work more efficient and easier.

\section{References}

1. R.K. Pekgöz, M.A. Gürel, M. Bilgehan, M. Kısa, Active Suspension of Cars Using Controller Optimized by Genetic Algorithm, Int. J. of Eng. and Appl. Sci. (IJEAS) 2, Issue 4 27-37, (2010)

2. http://ctms.engin.umich.edu/CTMS/index.php?exam ple $=$ Suspension\&section $=$ SystemModeling, (retrieved on 25.11.2017)

3. J. Jantzen, Foundations of fuzzy control, Chichester, England; Hoboken, NJ: John Wiley \& Sons, 2007.

4. N. E. I. S. U. Manual, NI Educational Laboratory Virtual Instrumentation Suite II Series (NI ELVISTM II Series) User Manual, (2009)

5. Ammar Alsabbagh, Péter T. SZEMES, Abdulkader Baki, Brushless DC Motor Modeling Using Bond Graph Method and Control using LabVIEW. (RIim) Recent Innovation in Mechatronics. Vol. 5, No 1, 2018 . 